Cahiers

Furles Droit Fondamentaux
Cahiers de la recherche sur les droits fondamentaux

$8 \mid 2010$

La liberté d'expression

\title{
Chronique de jurisprudence de la Cour interaméricaine des Droits de l'homme
}

Marie Rota

\section{(2) OpenEdition}

\section{Journals}

Édition électronique

URL : https://journals.openedition.org/crdf/6197

DOI : $10.4000 /$ crdf.6197

ISSN : 2264-1246

Éditeur

Presses universitaires de Caen

Édition imprimée

Date de publication : 20 décembre 2010

Pagination : 163-176

ISBN : 978-2-84133-367-7

ISSN : 1634-8842

\section{Référence électronique}

Marie Rota, "Chronique de jurisprudence de la Cour interaméricaine des Droits de l'homme ", Cahiers de la recherche sur les droits fondamentaux [En ligne], 8 | 2010, mis en ligne le 08 octobre 2020, consulté le 15 novembre 2022. URL : http://journals.openedition.org/crdf/6197 ; DOI : https://doi.org/10.4000/ crdf.6197 


\section{Chronique de jurisprudence de la Cour interaméricaine des Droits de l'homme ${ }^{1}$}

I. Les libertés physiques

A. Droit à la vie (article 4)

1. Droit à la vie et peine de mort

2. Droit à la vie et contexte de violence à l'égard des femmes

B. Le droit à la protection de la vie privée, de l'honneur et de la dignité de la personne (article 11)

II. La liberté de pensée et d'expression (article 13)

A. La liberté de pensée et d'expression: une "pierre angulaire de l'existence même d'une société démocratique"

B. Les obligations à la charge de l'État en vue de protéger la liberté de pensée et d'expression

C. L'examen des restrictions apportées à la liberté de pensée et d'expression

III. Les libertés relationnelles

A. La liberté d'association (article 16)

B. Les droits politiques (article 28)

IV. Les droits économiques, sociaux et culturels (article 26) et le droit à la propriété privée (article 21)

En 2009, la Cour a adopté 26 résolutions relatives aux mesures provisoires, 70 relatives à la supervision de l'exécution de trois de ses arrêts ${ }^{2}$, et 18 décisions au contentieux, dont 4 portent sur l'interprétation de décisions antérieures ${ }^{3}$. Les autres portent sur «les exceptions préliminaires, le fond et les réparations », la Cour ayant pris l'habitude d'examiner ces trois aspects dans un arrêt unique, pour des raisons d'économie de procédure. Cette tendance se confirme en 2009, deux décisions venant faire exception à ce principe ${ }^{4}$.

1. Chronique réalisée par Marie Rota, doctorante au CRDFED (Université de Caen Basse-Normandie), attachée temporaire d'enseignement et de recherche à l'Université du Havre, membre associé du GREDFIC (Université du Havre), du CEDIN (Centro de Direito Internacional - Brésil) et membre du GRIB (Groupe de recherche interdisciplinaire sur le Brésil).

2. Disponibles sur: www.corteidh.or.cr.

3. Cour IDH, Ticona Estrada et autres c. Bolivie, interprétation, $1^{\text {er }}$ juillet 2009, série C, $\mathrm{n}^{\circ} 199$; Cour IDH, Valle Jaramillo et autres c. Colombie, interprétation, 7 juillet 2009, série C, $\mathrm{n}^{\circ} 201$; Cour IDH, Escher et autres c. Brésil, interprétation, 20 novembre 2009, série C, $\mathrm{n}^{\circ} 208$; Cour IDH, Acevedo Buendía et autres ("Cesantes y Jubilados de la Contraloría») c. Pérou, interprétation, 24 novembre 2009, série C, $\mathrm{n}^{\circ} 210$.

4. Cour IDH, Kawas Fernández c. Honduras, fond, réparations et coûts, 3 avril 2009, série C, n 196 ; Cour IDH, Barreto Leiva c. Venezuela, fond, réparations et coûts, 17 novembre 2009, série C, $\mathrm{n}^{\circ} 206$. 
En outre, seule une décision est examinée suite à une reconnaissance de responsabilité, qui plus est uniquement partielle ${ }^{5}$. Cette année marque alors une rupture puisque, comme on l'a remarqué lors de notre précédente chronique, les reconnaissances étatiques de responsabilité étaient beaucoup plus nombreuses ${ }^{6}$. Ceci s'explique certainement par la nature du contentieux que la Cour a eu à traiter, qui recouvre des sujets beaucoup plus vastes que les classiques violations massives de droit. Bien que les questions traditionnelles de disparitions forcées ou de massacres se sont encore posées, elle a eu à traiter de la conformité à la Convention interaméricaine relative aux Droits de l'homme ${ }^{7}$ de pratiques nouvelles comme celle des écoutes téléphoniques.

On se contentera, dans le cadre de cette chronique, de traiter uniquement de la protection juridictionnelle des droits tels que consacrés par la Convention, sans revenir sur les questions de compétences ou de réparations, la Cour ne faisant que réaffirmer sa position à leur égard ${ }^{8}$. On a, de plus, sélectionné uniquement les droits qui ont fait l'objet de nouveaux développements ou qui n'avaient, jusque-là, pas encore été présentés dans le cadre de cette chronique. On commencera alors par aborder les libertés physiques (I), suivies de la liberté de pensée et d'expression (II), des libertés relationnelles (III) et, enfin, des droits économiques, sociaux et culturels et du droit à la propriété privée (IV).

\section{Les libertés physiques}

On abordera successivement l'analyse que fait la Cour de ces libertés physiques que sont le droit à la vie (A), et le droit à la protection de la vie privée, de l'honneur et de la dignité de la personne (B).

\section{A. Droit à la vie (article 4)}

Trois États ont été, en 2009, reconnus responsables de la violation du droit à la vie : la Barbade, le Mexique et le Pérou. Ces deux derniers ont en effet été condamnés suite aux disparitions forcées des victimes ${ }^{9}$, la Cour ayant appliqué une fois encore son "mécanisme déductif» présenté dans notre précédente chronique ${ }^{10}$. Mais le Mexique s'est également rendu responsable de cette violation dans le cadre d'un «contexte de violence à l'égard des femmes » (2) et la Barbade du fait de l'application à la victime de sa législation relative à la peine de mort (1).

\section{Droit à la vie et peine de mort}

Dans l'affaire Dacosta Cadogan c. Barbade ${ }^{11}$, la Cour interaméricaine a eu l'occasion de se prononcer sur la législation barbadienne relative à la peine de mort ${ }^{12}$. Elle rappelle alors la teneur de l'article 4.2 de la Convention qui autorise cette peine dans les États qui ne l'ont pas aboli, et conclut qu'elle n'est donc pas "per se incompatible avec la Convention» $(\$ 47)$. Elle souffre néanmoins de limites très strictes. Elle ne peut, tout d'abord, s'appliquer qu'aux crimes de droit commun les plus graves, à l'exclusion des infractions politiques ${ }^{13}$. La peine doit, ensuite, être individualisée « en fonction des caractéristiques de l'infraction, de [l'ampleur de] la participation du défendeur [à la commission de cette infraction] et de son degré de culpabilité ${ }^{14}$. Enfin, elle ne peut être prononcée que si elle satisfait au respect d'un certain nombre de garanties procédurales dont le respect doit être strictement observé ${ }^{15}$.

Elle a, en outre, considéré que les termes « arbitraire» et « délits les plus graves », employés dans les articles 4.1 et 4.2 respectivement, rendent incompatibles les dispositions de droit interne induisant une automaticité de la peine $^{16}(\$ 48)$. Elle insiste enfin sur le fait que les dispositions conventionnelles relatives à la peine de mort « doivent s'interpréter à la lumière du principe pro persona » dans la mesure où elles « imposent des restrictions ayant pour but de limiter rigoureusement l'application et la portée de la peine de mort, de façon à ce qu'ils se réduisent jusqu'à sa disparition totale ${ }^{17}(\$ 49)$.

Ces principes généraux rappelés, elle les confronte ensuite à l'espèce, en examinant dans un premier temps si la peine de mort est uniquement applicable aux délits les plus graves. De ce point de vue, elle se réfère à sa jurisprudence Boyce et autres, dans laquelle elle avait déjà affirmé que la législation barbadienne ne respectait pas

5. Cour IDH, Masacre de las Dos Erres c. Guatemala, exceptions préliminaires, fond, réparations et coûts, 24 novembre 2009 , série C, $\mathrm{n}^{\circ} 211$.

6. M. Rota, "Chronique de jurisprudence de la Cour interaméricaine des Droits de l'homme, septembre-décembre 2008 ", Cahiers de la recherche sur les droits fondamentaux, $\mathrm{n}^{\circ} 7$, L'universalisme des droits en question(s). La Déclartion universelle des Droits de l'homme, 60 ans après, 2009, p. 189-198, ciaprès «notre précédente chronique».

7. Ci-après «la Convention».

8. Cf. notre précédente chronique, p. 191-193 et 198.

9. Cour IDH, Radilla Pacheco c. Mexique, exceptions préliminaires, fond, réparations et coûts, 23 novembre 2009, série C, $\mathrm{n}^{\circ} 209$; Cour IDH, Anzualdo Castro c. Pérou, exceptions préliminaires, fond, réparations et coûts, 22 septembre 2009, série C, nº 202.

10. Cf. notre précédente chronique, p. 195 ; J. Benzimra-Hazan, «Disparitions forcées de personnes et protection du droit à l'intégrité : la méthodologie de la Cour interaméricaine des Droits de l'homme», Revue trimestrielle des Droits de l'homme, ${ }^{\circ}$ 47, juillet 2001, p. 765-796.

11. Cour IDH, Dacosta Cadogan c. Barbade, exceptions préliminaires, fond, réparations et coûts, 24 septembre 2009, série C, $n^{\circ} 204$.

12. Elle avait déjà eu à traiter de cette législation dans sa Cour IDH, Boyce et autres c. Barbade, exceptions préliminaires, fond, réparations et coûts, 20 novembre 2007 , série $\mathrm{C}, \mathrm{n}^{\circ} 169$, à laquelle elle renvoie régulièrement dans cette affaire.

13. Cour IDH, Hilaire, Constantine et Benjamin et autres c. Trinité-et-Tobago, fond, réparations et coûts, 21 juin 2002, série C, $\mathrm{n}^{\circ} 94, \$ 106$.

14. Ibid., $\$ 103-108$.

15. Cour IDH, Fermín Ramírez c. Guatemala, fond, réparations et coûts, 20 juin 2005, série C, $n^{\circ} 126, \$ 79$.

16. Cour IDH, Hilaire, Constantine et Benjamin et autres c. Trinité-et-Tobago, $\$ 103,106,108$.

17. Cour IDH, Hilaire, Constantine et Benjamin et autres c. Trinité-et-Tobago, $\$ 99$. 
cette condition. L'État ayant fait application de cette législation à la victime est donc reconnu responsable de la violation de l'article 4.2 de la Convention ( $\$ 50-52)$.

Elle s'attarde ensuite sur le « caractère arbitraire» de la peine de mort induite de son automaticité, prévue par le droit interne ${ }^{18}$. En effet, d'après la législation interne, toute personne ayant commis un homicide est automatiquement condamnée à mort, ce qui empêche les juges de procéder à une individualisation de la peine $(\$ 54)$. Dès lors, et même si elle peut solliciter une amnistie, elle se trouve dans l'impossibilité de s'adresser à un «tribunal compétent », tel que prévu par l'article 4.2 de la Convention, "pour déterminer si la peine de mort est la peine appropriée au cas d'espèce, conformément à la législation interne et à la Convention» $(\$ 56)$. La Cour en conclut donc qu'elle est contraire à l'article 4.1 de la Convention qui interdit la privation arbitraire du droit à la vie (\$57).

La Barbade est donc reconnue responsable de la violation des articles 4.1 et 4.2 de la Convention, lus conjointement avec son article 1. Elle est également responsable de la violation de l'article 2, qui lui impose non seulement d'adopter et de maintenir les mesures législatives nécessaires pour garantir l'exercice des droits consacrés mais aussi de ne pas promulguer des lois qui empêchent leur libre exercice $(\$ 68)^{19}$. Or, même si la Barbade s'est engagée à modifier sa législation et même sa Constitution pour les mettre en conformité avec la Convention (suite à la décision Boyce et autres, notamment), en l'espèce, les dispositions litigieuses ont bien été appliquées à la victime. L'État doit donc également être condamné sur ce fondement.

\section{Droit à la vie et contexte de violence à l'égard des femmes}

Dans l'affaire González et autres c. Mexique ${ }^{20}$, la Cour s'attarde sur la violation du droit à la vie de trois femmes, s'inscrivant dans un «contexte de violence à l'égard des femmes », connu de l'État. La Cour procède ici à une lecture combinée de l'article 4.1 de la Convention avec la Convention interaméricaine sur la prévention, la sanction et l'élimination de la violence contre la femme ${ }^{21}$, de laquelle elle déduit que la traditionnelle obligation de prévention des atteintes au droit à la vie à la charge de l'État se trouve renforcée à l'égard des femmes (\$258). Néanmoins, elle ne reconnaît pas que l'État ait failli à cette obligation positive, dans la mesure où il a adopté tout un panel de dispositions ayant justement pour objet de la mettre en œuvre. La Cour rappelle en effet que cette obligation de prévention ne pouvait engendrer une responsabilité illimitée de l'État (\$280 et 282 ).

Il n'a, en revanche, pas respecté son obligation procédurale - «obligation de moyen plus stricte» que la première - à savoir mener une enquête rapide et effective, dans la mesure où il a agi trop tardivement pour pouvoir identifier les responsables $(\$ 284)$. L'État a donc violé l'article 4.1 de la Convention lu conjointement à la fois avec son article 1.1 et l'article 7.b.y.c de la Convention de Belém.

On constate ici que la Cour attache une importance toute particulière à cette obligation procédurale découlant de l'article 4.1 de la Convention, et fondée sur sa lecture combinée avec l'article 1.1 selon lequel « Les États parties s'engagent à respecter les droits et libertés reconnus dans la présente Convention et à en garantir le libre et plein exercice à toute personne relevant de leur compétence». Or, dans cette même affaire, la Cour va également reconnaître le Mexique responsable de la violation des articles 8.1, 25.1 (relatifs au droit à un recours effectif et à un procès équitable) et 1.1, lus de manière conjointe ${ }^{22}$. On peut donc en conclure que la Cour distingue deux obligations d'enquête : celle émanant du volet procédural de l'article 4 - et du fait de la violation de ce droit, même par des particuliers - et celle fondée sur la combinaison des articles 8, 25 et 1.1 de la Convention, s'attachant cette fois-ci à la commission d'un acte illégal du point de vue du droit interne. Il y a là une véritable évolution, puisque la jurisprudence antérieure ne dégageait qu'une obligation d'enquête sur ce dernier fondement. Ce sujet a d'ailleurs fait l'objet de vives controverses doctrinales entre les deux anciens présidents de la Cour, Antônio Augusto Cançado Trindade ${ }^{23}$, d'une part, et Cecilia Medina Quiroga ${ }^{24}$, d'autre part, cette dernière ayant toujours plaidé pour la reconnaissance de ces deux obligations d'enquêtes distinctes ${ }^{25}$. Cette «bataille» semble donc avoir été remportée par la juge chilienne.

18. À cet égard, la Cour considère que l'automaticité de la peine de mort pour un crime particulier aboutit à ce que l'on refuse de reconnaitre la singularité des personnes reconnues coupables d'un tel crime, dans la mesure où cela revient à «traiter [ces] personnes non pas comme des êtres humains uniques, mais comme membres d'une masse anonyme, ne présentant pas de différence, et sujette à l'imposition aveugle de la peine de mort» ( $\$ 54)$; Cour IDH, Hilaire, Constantine et Benjamin et autres c. Trinité-et-Tobago, $\$ 105$.

19. Cour IDH, Castillo Petruzzi et autres c. Pérou, fond, réparations et coûts, 30 mai 1999, série C, $\mathrm{n}^{\circ} 52, \$ 207$.

20. Cour IDH, González et autres ("Campo Algodonero») c. Mexique, exceptions préliminaires, fond, réparations et coûts, 16 novembre 2009 , série C, $\mathrm{n}^{\circ}$ 205, ci-après « affaire González et autres c. Mexique».

21. Convention adoptée à Belém do Pará (Brésil) le 9 juin 1994, et entrée en vigueur le 5 mars 1995, ci-après «la Convention de Belém ».

22. Affaire González et autres c. Mexique, $\$ 388$.

23. Juge à la Cour interaméricaine de 1994 à 2006 et président de cette même Cour de 1999 à 2003.

24. Juge à la Cour interaméricaine de 2004 à 2009 et présidente de cette même Cour de 2008 à 2009

25. Voir à cet égard les ouvrages suivants: C. M. Quiroga, La Convención Americana: Teoría y jurisprudencia : vida, integridad personal, libertad personal, debido proceso y recurso judicial, Santiago, Universidad de Chile, Facultad de Derecho, Centro de Derechos Humanos, 2005 ; C. M. Quiroga et C. N. Rojas, Sistema interamericano de derechos humanos : introducción a sus mecanismos de protección, Santiago, Universidad de Chile, Facultad de Derecho, Centro de Derechos Humanos, 2007, mais aussi ses opinions dissidentes sous les affaires Cour IDH, 19 Comerciantes c. Colombie, fond, réparations et coûts, 5 juillet 2004, série C, $\mathrm{n}^{\circ}$ 109; Cour IDH, Comunidad Moiwana c. Suriname, exceptions préliminaires, fond, réparations et coûts, 15 juin 2005, série C, $\mathrm{n}^{\circ} 124$; Cour IDH, Gómez Palomino c. Pérou, fond, réparations et coûts, 22 novembre 2005, série C, $\mathrm{n}^{\circ} 136$. 


\section{B. Le droit à la protection de la vie privée, de l'honneur et de la dignité de la personne (article 11)}

Comme mentionné en introduction, la Cour interaméricaine a eu à traiter d'un aspect fort novateur: la régularité d'écoutes téléphoniques au regard du droit à la vie privée. Dans l'affaire Tristán Donoso c. Panamá ${ }^{26}$, tout d'abord, la victime, M. Santander Tristán Donoso, avocat, était, à l'époque des faits, employé comme conseiller juridique pour l'Église catholique. À la demande de l'évêque de Colón, il a accepté de défendre M. Walid Zayed, placé en détention provisoire dans le cadre d'une procédure pénale menée contre lui pour infraction de blanchiment d'argent. C'est dans ce cadre qu'il a été mis sur écoute sur ordre du procureur, ce dernier ayant par la suite divulgué les enregistrements à l'Église et au Collège national des avocats du Panamá ${ }^{27}$.

Dans l'affaire Escher et autres c. Brésil ${ }^{28}$, cinq membres de deux associations (ADECON et COANA) ayant des relations avec le Mouvement des travailleurs ruraux sans terre $^{29}$ (ci-après le MST) avec qui elles partagent l'objectif de promouvoir une réforme agraire au Brésil ${ }^{30}$, ont été mis sur écoute, après autorisation du juge. Néanmoins, si la première écoute a fait l'objet d'une demande motivée du maire, suspectant la participation de certains membres d'ADECON à des actions et pratiques criminelles prétendument menées par le MST, il n'en a pas été le cas pour la seconde, concernant la mise sur écoute des membres de COANA. De plus, si cette dernière a bien été notifiée au ministère public, garantie prévue par le droit brésilien, la mise sur écoute des membres d'ADECON ne l'a pas été. Enfin, les enregistrements de ces conversations ont, par la suite, été transmis à la presse.

Ces deux affaires sont l'occasion pour la Cour de rappeler le champ d'application de cet article 11 de la
Convention, qui interdit l'ingérence arbitraire ou abusive dans la vie privée des personnes, mais qui protège également d'autres domaines: la vie familiale, le domicile et les correspondances ${ }^{31}$. Elle rappelle ensuite sa jurisprudence Masacres de Ituango ${ }^{32}$, dans laquelle elle avait déjà affirmé que la violation de ce droit pouvait provenir de l'action soit des tiers, octroyant ainsi un certain effet horizontal à cette disposition, soit de l'autorité publique. Enfin, elle affirme que si les conversations téléphoniques ne sont pas visées expressément par cet article 11, il s'agit d'une "forme de communication » qui, au même titre que la correspondance, est inclue dans le champ de protection du droit à la vie privée ${ }^{33}$. Elle en a d'ailleurs une vision extensive puisqu'elle prend le soin de préciser que ce droit protège les conversations téléphoniques tant privées que professionnelles, et qu'elle englobe non seulement le contenu de la conversation, mais aussi tout le processus de communication (heure et durée de l'appel, destinataire, etc. $)^{34}$. Elle insiste enfin sur le devoir de protection attaché à ce droit en affirmant que l'État doit "prendre un engagement encore plus ferme pour s'adapter [à la modernité] » et faire face aux risques accrus d'atteinte du fait du développement des nouvelles technologies ${ }^{35}$.

Elle rappelle néanmoins qu'il n'est pas absolu ${ }^{36}$, et fait une lecture a contrario de l'article 11.2 de la Convention qui dispose que « Nul ne peut être l'objet d'ingérences arbitraires ou abusives dans sa vie privée, dans la vie de sa famille, dans son domicile ou sa correspondance, ni d'attaques illégales à son honneur et à sa réputation » ${ }^{37}$.

Elle considère, par conséquent, qu'il autorise l'État à le restreindre dans la mesure où ces ingérences ne sont pas abusives ou arbitraires. Elles ne sont donc compatibles avec la Convention que si elles sont prévues par la loi, si elles poursuivent un but légitime et sont «nécessaires dans une société démocratique ${ }^{38}$, cette dernière expression

26. Cour IDH, Tristán Donoso c. Panamá, exceptions préliminaires, fond, réparations et coûts, 27 janvier 2009, série C, $\mathrm{n}^{\circ} 193$, ci-après «affaire Tristán Donoso c. Panamá».

27. Équivalent panaméen du Barreau.

28. Cour IDH, Escher et autres c. Brésil, exceptions préliminaires, fond, réparations et coûts, 6 juillet 2009, série C, n 200, ci-après « affaire Escher et autres c. Brésil».

29. Ce mouvement a d'ailleurs été reconnu comme étant victime d'une violation des articles 8 et 25 de la Convention par la Cour dans l'affaire Cour IDH, Garibaldi c. Brésil, exceptions préliminaires, fond, réparations et coûts, 23 septembre 2009, série C, n 203, \$138. En l'espèce, la Cour ressent le besoin d'exprimer «sa préoccupation" au sujet de cette durée excessive et des irrégularités de procédure à l'égard de membres d'un "groupe considéré comme vulnérable », à savoir les membres du MST. Or, «l’impunité rend propice la répétition chronique de violations de Droits de l’homme 》 (\$141), et elle semble ainsi inviter le Brésil à y mettre fin au plus vite mais aussi l'avertir de la protection renforcée dont bénéficient les membres de ce groupe, qualifié de «vulnérable».

30. Voir, à ce sujet, la récente thèse de la sociologue S. Bleil, Engagement corps et âmes. Vies et luttes des Sans Terre dans le Sud du Brésil, Thèse de doctorat, R. M. Lagrave (dir.), EHESS, 2009, ou, pour un bref aperçu de la situation critique de ce mouvement pacifique qu'est le MST: S. Bleil, «Le mouvement des “sans terre". Naissance et développement d'une lutte populaire pour la réforme agraire au Brésil », Latitudes, n 23, 2005, p. 14-18.

31. Affaire Tristán Donoso c. Panamá, $\$ 55$; affaire Escher et autres c. Brésil, \$113.

32. Cour IDH, Masacres de Ituango c. Colombia, exceptions préliminaires, fond, réparations et coûts, $1^{\text {er }}$ juillet 2006, série C, $n^{\circ} 148, \$ 193$ et 194 .

33. Affaire Tristán Donoso c. Panamá, $\$ 55$; affaire Escher et autres c. Brésil, $\$ 114$. Elle justifie cette approche en faisant référence à la jurisprudence de la CEDH : Cour EDH, Klass et autres c. Allemagne, 6 septembre 1978, requête n ${ }^{\circ}$ 5029/71, \$29; Halford c. Royaume-Uni, 27 mai 1997, requête n ${ }^{\circ} 20605 / 92$, $\$ 44$; Cour EDH, Amann c. Suisse, 16 février 2000, requête n ${ }^{\circ}$ 27798/95, \$ 44; Cour EDH, Copland c. Royaume-Uni, 13 mars 2007 , requête ${ }^{\circ}$ 62617/oo, \$ 41.

34. Affaire Escher et autres c. Brésil, $\$ 114$.

35. Ibid., $\$ 115$.

36. Affaire Tristán Donoso c. Panamá, $\$ 56$; affaire Escher et autres c. Brésil, $\$ 116$.

37. La Cour prend le soin de distinguer ces deux derniers termes : alors que l'honneur « renvoie à l'estime de soi et de sa valeur propre ", la réputation renvoie «à l'opinion qu'ont les autres d'une personne» : affaire Tristán Donoso c. Panamá, \$ 57; affaire Escher et autres c. Brésil, \$117. La Cour rappelle encore cette distinction dans l'affaire González et autres c. Mexique, $\$ 444$.

38. Affaire Tristán Donoso c. Panamá, \$116; affaire Escher et autres c. Brésil, \$129. 
imposant qu'elles respectent les exigences d'adéquation (idoneidad), de nécessité et de proportionnalité ${ }^{39}$.

Or, d'après la Cour, l'interception et l'enregistrement des conversations téléphoniques d'une part, et la divulgation des écoutes téléphoniques ${ }^{40}$, d'autre part, sont des restrictions à ce droit. Elle va donc vérifier si elles répondent bien aux critères dégagés.

Concernant l'interception et l'enregistrement des conversations téléphoniques, et avant de procéder à l'examen des critères de compatibilité, la Cour cherche tout d'abord à déterminer la responsabilité étatique. Pour ce faire, elle vérifie qui est à l'origine des écoutes et, plus particulièrement, si elles sont le fait d'un agent de l'État, ce qui n'est pas prouvé dans la première affaire ${ }^{41}$, mais l'est dans la seconde ${ }^{42}$.

Elle recherche alors uniquement dans l'affaire Escher et autres c. Brésil si cette restriction était prévue par la loi, ce terme «loi » étant entendu «dans son sens formel et matériel ${ }^{43}$, et cette norme devant être suffisamment précise pour l'encadrer strictement $(\$ 131)$. Or, la Cour constate que les écoutes téléphoniques étaient bien prévues par la législation interne, mais, qu'en l'espèce, elle n'avait pas été respectée $(\$ 133-146)$. La restriction n'est donc pas compatible à la Convention et le Brésil est reconnu responsable de la violation de l'article 11 de la Convention, ses agents ayant procédé à une interception et un enregistrement de conversations téléphoniques sans y être autorisés.

Elle procède ensuite à la même démarche pour la divulgation des conversations téléphoniques et recherche une origine étatique potentielle. Dans la première affaire, les enregistrements avaient été transmis par l'ancien procureur général (agent étatique) à l'Église (employeur de la victime) et au Collège national des avocats du Panamá. Dans la seconde affaire, la Cour constate qu'une partie des enregistrements ont été transmis à une chaîne télévisée par un "agent étatique non identifié », et l'autre partie diffusée par l'ancien secrétaire de sécurité à des journalistes lors d'une conférence de presse. Le Panamá et le Brésil sont donc bien à l'origine de ces restrictions et pourront donc être reconnus responsables s'ils ne respectent pas les critères de compatibilité dégagés.

Les juges de San José constatent tout d'abord que les deux États avaient bien mis en place une législation autorisant la divulgation de conversations téléphoniques dans certains cas et ils recherchent alors si ces législations ont bien été respectées par les agents étatiques ayant procédé à ces divulgations.

Pour ce qui est de la loi panaméenne, elle n'autorisait le procureur, s'il estimait que cet enregistrement prouvait la participation des victimes à des actes criminels, qu'à faire un rapport, conduisant à une enquête, et c'est seulement dans le cadre strict de cette procédure pénale qu'une divulgation des enregistrements pouvait être autorisée. Par conséquent, le procureur ne pouvait en aucun cas les transmettre à l'Église en dehors de cette procédure. De la même façon, s'il présumait une atteinte potentielle à l'éthique de la profession d'avocat, il devait, selon la loi en vigueur, déposer une plainte au Tribunal de l'Honneur du Collège national des avocats, qui aurait alors procédé à une enquête, dans le cadre de laquelle il aurait pu alors transmettre les enregistrements. La divulgation des conversations téléphoniques est donc illégale, et ne peut être considérée comme une restriction compatible avec l'article sur le droit à la vie privée $(\$ 79-81)$. La Cour relève, en outre, qu' elle est constitutive d'une atteinte au droit à l'honneur et à la réputation de la victime, en ce qu'elle aboutissait à jeter un doute sur sa probité, ayant des répercussions inévitables sur l'opinion qu'avaient de lui l'Église et le Collège national des avocats $(\$ 82)$. Le Panamá est alors reconnu responsable d'une violation des droits à la vie privée et à l'honneur tels que protégés par les articles 11.1 et 11.2 de la Convention américaine, lus en relation avec l'article $1.1(\$ 83)$.

Pour ce qui est de la loi brésilienne, ensuite, la Cour constate que lorsque des écoutes téléphoniques s'inscrivent dans le cadre d'une enquête criminelle, pour laquelle elles sont autorisées, elles sont protégées par le secret de l'instruction. Elle relève en outre que, toujours d'après la loi brésilienne, le fait de «rompre un secret de justice, sans autorisation judiciaire et dans des objectifs contraires à la loi » est constitutif d'un crime $(\$ 160)$. La Cour en profite alors pour souligner l'importance qu'elle accorde au «secret des conversations téléphoniques interceptées au cours d'une enquête criminelle » qui est : « un devoir d'État: a) nécessaires pour protéger la vie privée des personnes soumises aux mesures de cette nature, b) relatives aux effets de recherche elle-même, et c) indispensables à la bonne administration de la justice » $(\$ 161)$.

Or, en l'espèce, cette divulgation n'avait pas été autorisée et était bien de nature à rompre ce secret $(\$ 162)$. La Cour estime par ailleurs que, même si elle aurait pu avoir pour but d'informer le public sur une question d'intérêt général, l'ancien Secrétaire de Sécurité aurait dû procéder à cette divulgation en passant par les voies légales, c'est-à-dire en sollicitant une autorisation de la part d'un tribunal $(\$ 163)$. La restriction au droit à la vie privée ayant pour but de donner plein effet à la liberté d'expression ne peut donc être admise que dans la mesure où elle est également prévue par la loi. Le Brésil est alors reconnu

39. Affaire Tristán Donoso c. Panamá, $\$ 56$; affaire Escher et autres c. Brésil, $\$ 129$.

40. La Cour suit ensuite la même démarche à l'égard de la divulgation des écoutes téléphoniques, qu'elle confronte au droit à la vie privée. Elle qualifie tout d'abord lesdites conversations téléphoniques de "privées ", en ce sens qu'aucun des deux interlocuteurs n'avait consenti à ce qu'elle soit connue des tiers (affaire Tristán Donoso c. Panamá, $\$ 75$; affaire Escher et autres c. Brésil, $\$ 158$ ). En outre, dans la première affaire, elles bénéficiaient d’une protection encore plus étendue du fait du secret professionnel auquel est soumis tout avocat.

41. Affaire Tristán Donoso c. Panamá, $\$ 61-66$.

42. Affaire Escher et autres c. Brésil, $\$ 125$.

43. Cour IDH, L'expression «loi » de l'article 30 de la Convention américaine relative aux Droits de l'homme, opinion consultative, 9 mai 1986, OC-6/86, série $\mathrm{A}, \mathrm{n}^{\circ} 6, \$ 27$ et 32 . 
responsable, tout comme le Panamá, d'une violation des articles 11.1 et 11.2 de la Convention américaine, lus en relation avec l'article $1.1(\$ 164)$.

\section{La liberté de pensée et d'expression (article 13)}

La Cour interaméricaine s'est prononcée sur la violation de l'article 13 de la Convention à quatre reprises : dans les affaires Tristán Donoso c. Panamá, Usón Ramírez c. Venezuela ${ }^{44}$, Ríos et autres $c$. Venezuela ${ }^{45}$ et Perozo et autres c. Venezuela ${ }^{46}$. Ces affaires ont permis à la Cour de réaffirmer l'importance de cette liberté (A), de rappeler les obligations à la charge de l'État (B) et sa méthodologie à l'égard des restrictions étatiques (C).

\section{A. La liberté de pensée et d'expression: une " pierre angulaire de l'existence même d'une société démocratique "}

Dans les quatre affaires, la Cour commence par rappeler la portée de ce droit, dégagée par sa jurisprudence antérieure ${ }^{47}$. Il englobe à la fois le droit « d'exprimer ses pensées ${ }^{48}$, « de chercher, de recevoir et de diffuser des informations et des idées de tout genre, et le droit de recevoir des informations et de connaître les pensées exprimées par d'autres ${ }^{49}$.

Il s'agit, de plus, d'un droit d'une importance toute particulière du fait de son rôle joué dans une «société démocratique ${ }^{50}$, notamment vis-à-vis du pluralisme, condition d'existence d'une telle société. L'exigence d'effectivité de la liberté d'expression n'en est d'ailleurs que plus forte, puisque sans elle, "les mécanismes de contrôle et de dénonciation des citoyens deviennent inopérants ", laissant place à un terrain propice au retour de régimes autoritaires $(\$ 105)$. La Cour renvoie à cet égard à l'affaire Herrera Ulloa $c$. Costa Rica ${ }^{51}$, dans laquelle elle avait déjà souligné cet aspect, ainsi que l'importance du rôle joué par cette liberté dans « la consolidation et dans la dynamique d'une société démocratique».

C'est pourquoi la Cour qualifie cette liberté de «pierre angulaire de l'existence même d'une société démocratique ${ }^{52}$, et insiste plus particulièrement sur ce rôle « en ce qui concerne des questions d'intérêt public ${ }^{53}$. Elle a par conséquent une conception aussi, voire plus large, que celle de Strasbourg ${ }^{54}$, puisqu' elle estime qu'elle doit être garantie: «non seulement à l'égard de la diffusion des informations et des idées qui sont accueillies avec faveur ou considérées comme inoffensives ou indifférentes, mais aussi en ce qui concerne celles qui sont désagréables pour l'État ou une fraction quelconque de la population ».

\section{B. Les obligations à la charge de l'État en vue de protéger la liberté de pensée et d'expression}

Du fait de cette importance toute particulière accordée à la liberté de pensée et d'expression, la Cour affirme que son exercice effectif implique une triple obligation à la charge de l'État. Une obligation négative renforcée tout d'abord, puisque l'État doit non seulement s'abstenir de porter atteinte à cette liberté, mais il doit également veiller à ce que « la participation des différents courants dans le débat public» soit équilibrée au nom du "pluralisme informatif » ${ }^{55}$. Dans ce cadre, il doit s'abstenir « d'agir d'une manière qui rende propice, encourage, favorise, ou accentue l'état de vulnérabilité » dans lequel se trouvent parfois certains journalistes. Il a ensuite l'obligation positive substantielle d'" adopter, lorsque cela est pertinent, les mesures nécessaires et raisonnables pour prévenir ou protéger les droits de ceux qui sont dans cette situation ». Enfin, il a, le cas échéant, l'obligation positive procédurale «d'enquêter sur des faits qui leur nuisent $»^{56}$.

Cette précision prend toute sa mesure face aux faits des espèces Ríos et autres $c$. Venezuela et Perozo et autres

44. Cour IDH, Usón Ramírez c. Venezuela, exceptions préliminaires, fond, réparations et coûts, 20 novembre 2009, série C, $\mathrm{n}^{\circ}$ 207, ci-après «affaire Usón Ramírez c. Venezuela».

45. Cour IDH, Ríos et autres c. Venezuela, exceptions préliminaires, fond, réparations et coûts, 28 janvier 2009, série C, $\mathrm{n}^{\circ}$ 194, ci-après « affaire Ríos et autres c. Venezuela».

46. Cour IDH, Perozo et autres c. Venezuela, exceptions préliminaires, fond, réparations et coûts, 28 janvier 2009, série C, $\mathrm{n}^{\circ}$ 195, ci-après « Perozo et autres c. Venezuela».

47. Cour IDH, La Colegiación Obligatoria de Periodistas (art. 13 et 29 de la Convention américaine relative aux Droits de l'homme), OC-5/85, 13 novembre 1985, série A, $\mathrm{n}^{\circ} 5, \$ 30$; Cour IDH, Ricardo Canese c. Paraguay, fond, réparations et coûts, 31 août 2004, série C, n ${ }^{\circ} 111, \$ 77$; Cour IDH, Kimel c. Argentine, 2 mai 2008, série $\mathrm{C}, \mathrm{n}^{\circ}$ 177, $\$ 53$. On peut à cet égard souligner la particularité et l'originalité de cette jurisprudence, reconnaissant le droit d'accéder à l'information, notamment celle détenue par l'État lorsqu'elle est « d'intérêt public », alors même que la Cour EDH ne reconnaît pas cet aspect.

48. Affaire Ríos et autres $c$. Venezuela, $\$ 104$; affaire Perozo et autres $c$. Venezuela, $\$ 105$.

49. Affaire Tristán Donoso c. Panamá, $\$ 109$; affaire Ríos et autres c. Venezuela, $\$ 104$; affaire Perozo et autres c. Venezuela, $\$ 115$.

50. Affaire Ríos et autres c. Venezuela, $\$ 105$; affaire Perozo et autres c. Venezuela ; affaire Tristán Donoso c. Panamá, $\$ 113$.

51. Cour IDH, Herrera Ulloa c. Costa Rica, exceptions préliminaires, fond, réparations et coûts, 2 juillet 2004, série C, $\mathrm{n}^{\circ} 107$.

52. Affaire Ríos et autres c. Venezuela, $\$ 105$; affaire Perozo et autres $c$. Venezuela; affaire Usón Ramírez c. Venezuela, $\$ 23$.

53. Affaire Ríos et autres $c$. Venezuela, $\$ 105$; affaire Perozo et autres $c$. Venezuela; affaire Usón Ramírez c. Venezuela, $\$ 23$; Cour IDH, La Colegiación Obligatoria de Periodistas, $\$ 70$.

54. Cour EDH, 7 décembre 1976, Handyside c. Royaume-Uni, requête nº 5493/72, 449.

55. Affaire Ríos et autres $c$. Venezuela, $\$ 106$; affaire Perozo et autres $c$. Venezuela, $\$ 117$.

56. Affaire Ríos et autres $c$. Venezuela, $\$ 106$; affaire Perozo et autres $c$. Venezuela, $\$ 107$. 
c. Venezuela. Elles concernent toutes deux des médias privés vénézuéliens - la «Compañía Anónima Radio Caracas Televisión » (ci-après RCTV) et la chaîne de télévision Globovisión - qui avaient eu un rôle actif dans la tentative de coup d'État dirigée contre le président Hugo Chávez de 2002. C'est suite à ces événements, que des menaces et actes de violence ont été dirigés à l'encontre de 20 et 44 personnes respectivement ${ }^{57}$, travaillant directement ou indirectement pour ces deux médias privés. Elles invoquent en outre des déclarations publiques de fonctionnaires, dirigées contre ces deux médias ${ }^{58}$, attisant la haine de certains à l'encontre de toute personne y travaillant.

Pour déterminer le degré de responsabilité étatique, elle procède en trois temps. Elle établit dans un premier temps le contexte dans lequel se sont produits les faits reprochés. En l'espèce, elle souligne l'existence d'un contexte de «forte polarisation et de troubles politiques et sociaux", alors que le Venezuela avait eu connaissance des situations menaçantes dans lesquelles se trouvaient les victimes (du fait de plaintes déposées par ces dernières $)^{59}$. Elle recherche ensuite quelle est l'intensité du devoir de protection et de prévention au regard de ce contexte spécifique. Dans les deux espèces, elle constate que les agents de l'État - qualifiés de «garants des Droits de l'homme» et d' "orateurs privilégiés dans une société démocratique » - ont à leur charge un « devoir de prudence spécifique» qui se trouve "accentué lors de conflits sociaux ${ }^{60}$. Enfin, elle constate que ce devoir n'a pas été accompli, puisque les propos tenus par ces fonctionnaires ont contribué à accentuer l'état de vulnérabilité dans lequel se trouvaient déjà les journalistes et employés de la RCTV et de la Globovisión ${ }^{61}$. L'État a donc failli à son obligation négative ci-dessus énoncée, puisque ses agents ont porté atteinte au droit des victimes de rechercher, recevoir et diffuser des informations, ces dernières se trouvant dans une situation d'intimidation, accentuée par leurs propos. Il a, en outre, failli à son obligation positive substantielle de prévenir des atteintes aux Droits de l'homme alors qu'il avait eu connaissance d'un tel risque ${ }^{62}$.
La Cour souligne enfin l'importance de l'obligation positive procédurale à la charge de l'État et considère la voie pénale comme la plus appropriée pour sanctionner des atteintes à la liberté de pensée et d'expression ${ }^{63}$. La Cour procède ensuite à un examen précis des procédures pénales internes empruntées par les victimes et reproche au ministère public de ne pas avoir ordonné d'enquête ex officio suite aux plaintes de certaines d'entre elles, sous prétexte qu'elles avaient saisi la mauvaise juridiction ${ }^{64}$. La Cour relève, de plus, qu'en ce qui concerne les autres victimes, ayant emprunté les voies de recours prévues, les procédures ont pris un retard conséquent (de deux à six ans selon les cas) et injustifié ${ }^{65}$. L'État a donc violé son obligation procédurale issue de la combinaison de l'article 13.1 avec l'article 1.1 de la Convention. Il est ici intéressant de noter que cette violation du volet procédural de l'article 13 est de nature à "absorber» celle de l'obligation d'enquête découlant de l'interprétation combinée des articles 8.1,25.1 et 1.1 de la Convention. En effet, les juges affirment dans les deux espèces que cette dernière obligation est analysée au travers de la première ${ }^{66}$ qu'ils font donc prévaloir sur la seconde.

\section{L'examen des restrictions apportées à la liberté de pensée et d'expression}

La Cour a dû apprécier la validité de restrictions apportées à la liberté d'expression dans les affaires Tristán Donoso c. Panamá et Perozo et autres c. Venezuela. Car si elle reconnaît une importance particulière à cette liberté, elle rappelle qu'elle n'est pas absolue puisque l'article 13.2 prévoit qu'elle peut faire l'objet de restrictions lorsqu'elle interfère avec d'autres droits garantis par la Convention. La Cour rappelle en effet que « chaque droit fondamental doit s'exercer dans le cadre du respect et de la sauvegarde des droits fondamentaux d'autrui ${ }^{67}$. Elle souligne ensuite le «rôle central» de l'État, qui devra chercher à établir les responsabilités et les sanctions qui sont nécessaires à la poursuite de ce but ${ }^{68}$. Elle rappelle enfin «l'importance de la liberté d'expression dans une société démocratique», ce qui implique de la part de l'État de

57. Entre 2001 et 2004 et entre octobre 2001 et août 2005 respectivement.

58. Il ressort en effet de ces déclarations que ces deux chaînes sont surnommées, avec deux autres, «les quatre cavaliers de l’apocalypse », et qu'elles sont régulièrement qualifiées de "fascistes ", d' « ennemis du peuple vénézuélien », d' « ennemis de la révolution ", et soupçonnées d'être impliquées « dans un complot terroriste», «dans une action déstabilisatrice contre le Venezuela, contre le peuple, contre les lois et contre la République », de « conspirer contre la Révolution", et d'avoir participé à ladite tentative de coup d'État de 2002 : affaire Ríos et autres $c$. Venezuela, $\$ 127$; affaire Perozo et autres c. Venezuela, $\$ 139$

59. Affaire Ríos et autres $c$. Venezuela, $\$ 121-131$; affaire Perozo et autres $c$. Venezuela, $\$ 132-143$.

60. Affaire Ríos et autres c. Venezuela, $\$ 139-142$; affaire Perozo et autres $c$. Venezuela, $\$ 151-154$

61. Ils sont, en outre, à l'origine de l'assimilation de ces personnes aux dirigeants et propriétaires des deux chaînes, alors même que tous n'adhèrent pas forcément à leur politique.

62. Affaire Ríos et autres $c$. Venezuela, $\$ 149$; affaire Perozo et autres $c$. Venezuela, $\$ 161$. L'on peut remarquer ici que la Cour refusera ensuite d'examiner si ces propos tenus par lesdits fonctionnaires constituent une restriction justifiée à cette liberté dans la mesure où ces faits viennent d'être examinés et condamnés sous le volet «obligations à la charge de l’État» : affaire Ríos et autres c. Venezuela, $\$ 341$; affaire Perozo et autres $c$. Venezuela, $\$ 369$.

63. Affaire Ríos et autres $c$. Venezuela, $\$ 282-285$; affaire Perozo et autres c. Venezuela, $\$ 298-299$.

64. Affaire Ríos et autres c. Venezuela, $\$ 304$; affaire Perozo et autres $c$. Venezuela, $\$ 321$.

65. Affaire Ríos et autres $c$. Venezuela, $\$ 311-329$; affaire Perozo et autres $c$. Venezuela, $\$ 330-359$

66. Affaire Ríos et autres c. Venezuela, $\$ 76$; affaire Perozo et autres c. Venezuela, $\$ 88$.

67. Elle fait ici implicitement référence aux articles 29 et 32 de la Convention, qui consacrent également cette approche, à l'égard de l'ensemble des dispositions de la Convention.

68. Cour IDH, Kimel, $\$ 75$ 
minimiser les restrictions à la circulation des informations et «d'équilibrer, dans la mesure du possible, la participation des différentes informations dans le débat public, promouvoir le pluralisme de l'information ${ }^{69}$. La Cour semble donc donner une portée maximale à la liberté de pensée et d'expression qui, dans une démarche de conciliation avec d'autres droits, prévaudra. Elle estime de surcroît qu'elle doit être encore davantage protégée lorsque les propos contestés s'insèrent dans un «débat public». La Cour confirme dès lors l'existence d'un seuil de protection différencié à l'égard de la liberté de pensée et d'expression, justifié par «l'intérêt public» porté à un certain type de pensées et d'expression ${ }^{70}$.

Son examen des restrictions à la liberté d'expression répond à une méthodologie très stricte, qu'elle rappelle dans les affaires étudiées. La Cour doit en effet, dans un premier temps, déterminer ce "seuil de protection» attaché aux propos en cause, pour s'attarder ensuite sur la validité de la restriction en cause en fonction de ce seuil.

Dans la première affaire, $M$. Tristán Donoso, avocat, a été condamné à une peine de dix-huit mois de prison pour délit de calomnie et d'injure suite à une conférence de presse qu'il a organisée et dans laquelle il a déclaré que l'ancien procureur l'avait mis sur écoute, avait enregistré une conversation téléphonique qu'il avait eue avec son client, puis avait divulgué les enregistrements de manière illégale. Or, la Cour estime que ces propos bénéficient d'un seuil élevé de protection, en ce qu'ils relèvent du "débat public». En effet, ils concernent «l'aptitude d'une personne au regard de l'exercice de fonctions publiques ou des actes commis par des fonctionnaires dans l'accomplissement de leur travail », présentant un intérêt tout particulier pour les citoyens d'un État. D'ailleurs, selon elle, tout représentant de l'État, en ayant accepté d'exercer des fonctions publiques, «s'est volontairement exposé » à de telles critiques.

Il en va de même dans la seconde affaire. En l'espèce, un militaire retraité a été condamné à cinq ans et six mois d'emprisonnement pour le crime «d'insulte contre les Forces armées nationales ", suite à des propos tenus lors d'une émission télévisée. Il était chargé d'apporter un éclairage sur un incendie qui s'était produit dans une caserne et a, dans ce cadre, implicitement suspecté les forces armées d'avoir utilisé de manière volontaire des lance-flammes comme moyen de sanction à l'encontre de soldats. Or, la Cour rappelle que les propos «ayant trait à l'exercice des fonctions institutionnelles de l'État jouissent d'une protection renforcée ${ }^{71}$, comme c'est le cas en l'espèce.

La Cour procède ensuite à un test de compatibilité de ces restrictions à la Convention, eu égard à ce seuil renforcé de protection, en recherchant si les exigences de «légalité », « adéquation », «nécessité » et "proportionnalité » sont respectées ${ }^{72}$.

Dans l'affaire Tristán Donoso c. Panamá, tout d'abord, la Cour constate que la peine de « calomnie », à laquelle a été condamnée la victime, était bien prévue à l'article 172 du Code pénal $(\$ 117)$.

Cette première exigence ayant été respectée, la Cour se livre ensuite à l'examen de son « adéquation» au but recherché. Sous cette appellation, la Cour va examiner deux choses : d'une part, si la finalité de la restriction est légitime; d'autre part, si la procédure adoptée est adéquate vis-à-vis de cet objectif. En l'espèce, le but de la restriction étant la protection de l'honneur et de la réputation d'autrui - droit lui-même protégé à l'article 11 de la Convention -, il est considéré comme légitime, compatible avec la Convention. La voie pénale est, en outre, considérée comme un moyen adéquat pour l'atteindre ${ }^{73}$ (\$118).

La troisième exigence, la «stricte nécessité », n'est en revanche pas respectée, eu égard d'une part à la nature des propos de la victime - qui n'a fait que divulguer des faits exacts ${ }^{74}$, à savoir que l'ancien procureur avait intercepté et enregistré une conversation téléphonique de manière illégale - et d'autre part à l'importance de ces propos au regard du débat public ci-dessus identifié ( $\$ 119-130)$. La Cour, sans avoir besoin de s'attarder sur la troisième exigence, en conclut que l'État a violé le droit à la liberté d'expression de M. Tristán Donoso, et par là même l'article 1.1 de la Convention ${ }^{75}$.

Dans l'affaire Perozo et autres $c$. Venezuela, la Cour va, de la même façon, rechercher si cette restriction à la liberté d'expression était compatible avec la Convention, en commençant par examiner si l'exigence de légalité est respectée. Elle constate que la sanction est bien prévue par le Code pénal militaire. Elle considère en revanche que la disposition en question ne définit pas de manière suffisamment précise les faits susceptibles d'être qualifiés de crime «d'insulte aux forces armées nationales » $(\$ 33)$. L'exigence de légalité n'étant pas respectée, elle en conclut que l'État a violé l'article 13 de la Convention ${ }^{76}$, lu conjointement avec l'article $1.1(\$ 34)$.

69. Affaire Ríos et autres $c$. Venezuela, $\$ 111$; affaire Perozo et autres $c$. Venezuela, $\$ 113$.

70. Cour IDH, Herrera Ulloa c. Costa Rica, $\$ 129$.

71. Ibid., $\$ 128$

72. Cour IDH, Kimel; ce principe est également rappelé dans l’affaire Usón Ramírez c. Venezuela, \$25. Il contredit la vision de Rodolfo Brenes Vargas qui affirmait suite à l'analyse d'affaires antérieures que «Cette exigence de proportionnalité n'est pas conçue comme une quatrième condition de conventionalité, mais comme corollaire de la "nécessité" ». Elle est en effet dorénavant une exigence à part entière. R. B. Vargas, "Liberté d'expression et droit pénal devant la Cour interaméricaine des Droits de l'homme ", Revue de science criminelle et de droit pénal, $\mathrm{n}^{\circ}$ 2, avril-juin $2007, \mathrm{p} .366$.

73. Cf. Cour IDH, Kimel, $\$ 71$.

74. La Cour estime en effet que la liberté d'expression protège tout propos insusceptible d'être "qualifié de vrai ou de faux ", ou, comme en l'espèce, des faits réels. Des propos relatant des faits erronés ne sont en effet pas protégés dans la mesure où ils peuvent porter atteinte au droit à l'honneur et à la réputation des tiers.

75. Elle ne considère en revanche que l'État n'a pas violé l'article 2 de la Convention, qui met à la charge des États une obligation de mise en conformité de leur droit interne avec cette dernière, dans la mesure où ladite peine ne découlait pas d'une défaillance normative ( $(131)$.

76. Mais aussi son article 9, qui protège le principe de légalité. 
Il apparaît dès lors assez surprenant que la Cour poursuive néanmoins son " test de compatibilité » en recherchant si la restriction choisie est " adéquate».

Comme dans l'affaire précédente, les juges de San José affirment dans un premier temps que la protection du droit à l'honneur ou à la réputation est un objectif légitime, même s'il concerne, en l'espèce, les forces armées $(\$ 42)^{77}$.

Elle examine ensuite le second aspect de cette exigence et recherche si la procédure pénale peut être considérée comme adéquate pour atteindre cet objectif légitime. Elle relève tout d'abord que la voie pénale peut être considérée comme adéquate lorsqu'il s'agit de restreindre certains droits, faisant ainsi allusion à l'affaire Tristán Donoso c. Panamá ${ }^{78}$. Mais elle vient préciser de surcroît que cet aspect ne saurait signifier que la voie pénale est systématiquement considérée comme remplissant de facto les exigences de nécessité et de proportionnalité. Cette précision peut paraître surabondante mais semble avoir été apportée pour distinguer cette exigence "d'adéquation» de la procédure choisie au but légitime et l'exigence de "nécessité» de cette même procédure face aux faits de l'espèce. Les juges, tout en réaffirmant leur position, éprouvent néanmoins quelques difficultés à distinguer ces deux aspects et se réfugient derrière le caractère «vague et ambigu » de la loi pénale, pour affirmer qu'elle n'était pas adéquate. La difficulté aurait pourtant pu être écartée, si la Cour avait clairement affirmé que la voie pénale remplissait bien cette exigence, mais qu'elle n'était pas nécessaire, voire disproportionnée. Mais cela aurait alors avoué le caractère superflu de la première exigence vis-à-vis de ces deux dernières.

Ce constat est encore renforcé lorsqu'on s'attarde sur l'examen auquel s'est livré la Cour de l'exigence de nécessité de la mesure adoptée. Elle précise en effet dans un premier temps qu'elle ne sera considérée comme nécessaire que si l'État ne pouvait justifier d'autres alternatives possibles, plus douces $(\$ 48)$. Opérant ainsi une sorte de contrôle maximum, la Cour relève que le droit pénal est le «moyen le plus restrictif et le plus sévère pour établir les responsabilités au sujet d'une conduite illicite ». Il ne doit donc être utilisé qu'en dernier recours $(\$ 49)$, et il en va de la légitimité de l'exercice du « pouvoir répressif de l'État dans une société démocratique ${ }^{79}$. La Cour n'interdit cependant pas à l'État d'utiliser la voie pénale pour sanctionner un abus de la liberté d'expression, mais insiste sur le fait que cette voie doit être «strictement nécessaire » aux faits d'espèce et sera donc soumise à un strict examen, au cas par cas $(\$ 50)$. Et, une fois encore, elle se réfugie derrière le caractère vague de la loi pénale, qui reflète, de ce simple fait, l'exercice abusif du «pouvoir répressif » de l'État; elle ne peut donc pas être considérée comme nécessaire $(\$ 51)$.

$\mathrm{Au}$ regard de ces développements, on ne peut que difficilement saisir comment différencier les deux critères «d'adéquation » et de «nécessité » de la mesure, si ce n'est que le premier s'apprécierait en fonction de l'objectif de la restriction (et donc en l'espèce de l'article 11) et le second en fonction du droit restreint (l'article 13).

On peut souligner la complexité du raisonnement de la Cour, qui, de surcroît, ne s'arrête pas à cette étape. Elle procède en effet à un examen de la dernière exigence, à savoir la stricte proportionnalité de la restriction. Dans ce cadre, elle rappelle sa jurisprudence antérieure selon laquelle une restriction n'est considérée compatible avec la Convention, que si «le sacrifice qui lui est inhérent n'est pas déraisonnable ou excessif par rapport aux avantages procurés » qui la justifient ${ }^{80}(\$ 55)$. La Cour se livre ici à une sorte de " contrôle du bilan coût-avantage», en utilisant trois critères : «elle devra analyser i) le degré de restriction du droit en cause [...] ii) l'importance de la garantie apportée au second droit, et iii) si la garantie de ce dernier justifie la restriction de ce premier ${ }^{81}$.

En l'espèce, elle estime que, eu égard à la nature de la procédure menée à son encontre, à savoir un procès pénal, qui plus est, militaire, ainsi qu'à tout un ensemble de conséquences inéluctables liées à la nature de la peine ${ }^{82}$, la restriction devait être considérée comme «véritablement grave» $(\$ 57)$. Elle reconnaît, de la même façon, que la protection du droit à l'honneur et à la réputation est de toute importance $(\$ 58)$, mais ne suffit pas à justifier ladite restriction du fait de deux aspects. Premièrement, du fait de la nature des propos relevant d'un « intérêt public notoire»; ensuite, du fait qu'il ne s'agit que de l'expression d'opinions, qui, «n'étant pas susceptibles d'être considérées comme vraies ou fausses" ne peuvent être sanctionnées ${ }^{83}$. La restriction est donc disproportionnée (\$60-63). La Cour conclut (enfin!) que la condamnation de la victime à une peine d'emprisonnement est une restriction à sa liberté d'expression non compatible à la Convention $(\$ 64)$.

La Cour se livre donc à un contrôle extrêmement poussé et ne se contente pas de s'arrêter à la constatation

77. Ils précisent néanmoins qu’elles ne peuvent être considérées, en tant que personne morale, comme titulaires de ce droit ( $\$ 39)$.

78. Cour IDH, Kimel, $\$ 76$; Cour IDH, Tristán Donoso c. Panamá, $\$ 118$.

79. Cette légitimité dépend de son exercice qui doit être «strictement nécessaire » pour protéger les «biens juridiques fondamentaux » contre les attaques les plus graves.

80. Cour IDH, Kimel, $\$ 84$.

81. "Para efectuar esta ponderación se debe analizar i) el grado de afectación de uno de los bienes en juego, determinando si la intensidad de dicha afectación fue grave, intermedia o moderada; ii) la importancia de la satisfacción del bien contrario, et iii) si la satisfacción de éste justifica la restricción del otro. En algunos casos la balanza se inclinará hacia la libertad de expresión et en otros a la salvaguarda del derecho a la honra et reputación» (affaire Usón Ramírez c. Venezuela, \$56).

82. La Cour relève la nature de la peine en elle-même, et ses conséquences, à savoir : « [...] cinq ans et six mois d'emprisonnement; l'enregistrement au casier judiciaire; la perte de revenu durant cette période d'emprisonnement; les restrictions dans la jouissance de l'exercice des droits inhérents à cette peine ; le fait d'être éloigné de sa famille et de ses proches ; le risque latent d'une éventuelle perte de liberté personnelle, et l'effet stigmatisant de la condamnation pénale prononcée à son encontre $[\ldots] »$.

83. Cour IDH, Kimel, \$ 93. La Cour renvoie également à l'arrêt de la Cour EDH, Lingens $C$. Autriche, 8 juillet 1986, requête ${ }^{\circ} 9815 / 82, \$ 46$. 
du non-respect d'une de ces exigences, qui aurait pourtant pu suffire à caractériser l'incompatibilité de la restriction à la Convention ${ }^{84}$.

\section{Les libertés relationnelles}

On étudiera sous ce titre la protection juridictionnelle de la liberté d'association (A) et des droits politiques (B), les deux facettes traditionnelles de ces libertés dites « relationnelles».

\section{A. La liberté d'association (article 16)}

Dans l'affaire Escher et autres c. Brésil, la Cour recherche si la mise sur écoute téléphonique (illégale) porte atteinte au droit protégé par l'article 16 de la Convention des membres des deux associations concernées ${ }^{85}$. Dans cette démarche, elle justifie son analyse limitée à la liberté d'association et son refus d'analyser cet aspect sous l'angle de la liberté de réunion, du fait de leur différence de champ d'application.

En effet, d'après la Cour, la liberté de réunion «ne nécessite pas la participation à une entité ou à une organisation » et manifeste un caractère beaucoup plus spontané, l'union pouvant poursuivre « des buts des plus divers, à partir du moment où ils sont pacifiques et conformes à la Convention» $(\$ 169)$.

Elle réaffirme ensuite la portée qu'elle donne à cet article 16 : «toute personne étant sous la juridiction des États parties a le droit de s'associer librement avec d'autres personnes, sans l'intervention des autorités publiques limitant ou interférant dans l'exercice de ce droit. Il s'agit donc du droit de se regrouper, dans l'optique de rechercher la réalisation commune d'un but licite, sans pression ou ingérences qui pourraient altérer ou dénaturer ladite finalité» $(\$ 170)$.

L'État a donc l'obligation négative de ne pas limiter ou interférer dans l'exercice de ce droit. Mais cet article implique également des obligations positives de sa part: obligation de prévention des atteintes à ce droit tout d'abord; obligation de protéger (de manière active) cette liberté ensuite; et, enfin, obligation procédurale de mener une enquête lorsque cette liberté a été violée - la Cour prenant le soin de préciser que ces obligations s'inscrivent également « dans la sphère des relations entre particuliers » $(\$ 171)$.

Ce droit peut néanmoins faire l'objet de restrictions, qui, pour être admissibles, doivent être prévues par la loi, poursuivre un but légitime, et être nécessaires dans une société démocratique $(\$ 173)$.

Après avoir rappelé ces principes généraux, la Cour les confronte avec les faits de l'espèce et considère que la mise sur écoute des membres d'une association (fondée sur l'appartenance à celle-ci) ne peut être considérée comme une restriction compatible, étant donné son caractère illégal d'une part, et le fait qu'elle n'ait pas été demandée dans le cadre d'une enquête d'autre part $(\$ 174-178)$. De plus, la divulgation des conversations a induit pour les membres de ces associations « des craintes et des conflits » ainsi que, pour les associations ellesmêmes, une atteinte à leur image et à leur crédibilité. L'État a donc interféré de manière illégale et illégitime dans l'exercice du droit à la liberté d'association des victimes et est donc responsable de la violation de ce droit, lu de manière conjointe avec l'article 1.1.

\section{B. Les droits politiques (article 28)}

Dans l'affaire Reverón Trujillo c. Venezuela ${ }^{86}$, les représentants de la victime, «juge provisoire ${ }^{87}$ illégalement démis de ses fonctions, reprochent à l'État d'avoir violé l'article $23-1-c^{88}$, du fait du traitement inégal qu'elle a subi quant à l'accès et au maintien dans ses fonctions. En effet, alors que les juges internes ont reconnu qu'elle avait été démise de ses fonctions de manière irrégulière, ils n'ont pas pour autant imposé à l'État une obligation de « réintégration de la victime» (\$140). La question qui se posait alors à la Cour était celle de savoir quelle était la portée de cette disposition et si elle incluait un éventuel droit d'être maintenu dans ses fonctions ${ }^{89}$.

Pour y répondre de manière positive, la Cour s'appuie sur sa jurisprudence antérieure ${ }^{90}$. Dans une affaire Apitz Barbera et autres ${ }^{91}$, elle avait en effet précisé que cet article 23.1.c n'établissait pas un droit à toute personne d'accéder aux fonctions publiques, mais que l'accès à ces fonctions devait répondre à «des conditions générales d'égalité ». La Cour considère cette exigence comme satisfaite dès lors que «les critères et les procédures de nomination, de promotion, de suspension et de licenciement

84. Dans l'affaire Tristán Donoso c. Panamá ci-dessus étudiée, la Cour s'arrête d'ailleurs à l'examen de l'exigence de nécessité, sans s'attarder sur celle de proportionnalité, cette première n'étant pas respectée.

85. Voir supra, sous le titre «Protection de la vie privée, de l'honneur et de la dignité de la personne».

86. Cour IDH, Reverón Trujillo c. Venezuela, exceptions préliminaires, fond, réparations et coûts, 30 juin 2009, série C, n 197, ci-après « affaire Reverón Trujillo c. Venezuela».

87. Expression employée par opposition aux «juges titulaires», qui bénéficient de la garantie d’inamovibilité.

88. Cet article dispose que « tous les citoyens doivent jouir des droits et facultés [...] d'accéder, à égalité de conditions générales, aux fonctions publiques de leur pays".

89. Cette question avait déjà été posée à la Cour dans l'affaire Tribunal constitutionnel $c$. Pérou, sans qu'elle n'y ait répondu, les victimes ayant en l'espèce été réintégrées dans leurs fonctions; elle relève alors uniquement que les victimes avaient bien eu accès dans des conditions d'égalité à ces fonctions (Cour IADH, Tribunal constitutionnel c. Pérou, fond, réparations et coûts, 31 janvier 2001, série C, $\mathrm{n}^{\circ} 71$, \$98-104).

90. Elle cite également les décisions du Comité des Droits de l'homme des Nations unies, par lesquelles il a interprété l'article 25.c. du Pacte international relatif aux droits civils et politiques comme englobant à la fois l'accès et le maintien dans les fonctions publiques.

91. Cour IDH, Apitz Barbera et autres ("Corte Primera de lo Contencioso Administrativo») c. Venezuela, exceptions préliminaires, fond, réparations et coûts, 5 août 2008 , série C, $\mathrm{n}^{\circ} 182$ 
[sont] raisonnables et objectifs» et que «les personnes ne font pas l'objet de discriminations » dans l'exercice de ce droit ${ }^{92}$. L'accès aux fonctions publiques dans des conditions d'égalité doit donc nécessairement être accompagné d'une protection effective du maintien dans lesdites fonctions $(\$ 138)$.

La Cour souligne ensuite l'importance qu'elle accorde à la fonction publique, qui constitue le prolongement de la politique gouvernementale, et à l'effectivité de ce droit ${ }^{93}$ (\$139). Or comme elle l'a déjà affirmé, la différence de traitement entre les juges titulaires et les juges provisoires n'obéit pas à un critère raisonnable conforme à la Convention ( $\$ 114-117$ et 121). Par conséquent, $\mathrm{M}^{\mathrm{me}}$ Reverón Trujillo a subi une inégalité de traitement arbitraire concernant son droit d'être maintenue dans l'exercice de fonctions publiques. L'État est donc reconnu responsable de la violation combinée des articles 23.1.c et 1.1 de la Convention.

\section{Les droits économiques, sociaux et culturels (article 26) et le droit à la propriété privée (article 21)}

Si, contrairement à la Convention de sauvegarde des Droits de l'homme et des libertés fondamentales, la Convention américaine relative aux Droits de l'homme contient expressis verbis une clause - l'article 26 - relative "au développement progressif des droits économiques, sociaux et culturels", la Cour s'est toujours montrée assez frileuse quant à la construction d'une jurisprudence protectrice de ces droits.

Ceci explique la retenue judiciaire de la Commission dans l'affaire Acevedo Buendía et autres c. Pérou ${ }^{94}$, qui n'a allégué que la violation de l'article 21, protégeant le droit à la propriété privée, suite à l'adoption et à l'application de dispositions législatives et réglementaires péruviennes ayant pour objet de diminuer le montant des retraites des victimes. La juridiction constitutionnelle interne avait invalidé ces dispositions, et ordonné à l'État de remettre les victimes en l'état, et donc de leur restituer les sommes dues. Or, les autorités ne se sont pas conformées à cette décision, et la Commission, tout comme les représentants des victimes, allèguent donc que leur droit de propriété a été violé.
Mais ces derniers ont de surcroît allégué « une violation du droit à la sécurité sociale, qui comprend le droit à une "retraite nivelée" »" ${ }^{95}$ "tel qu'il est garanti par l'article 26 de la Convention », en ce qu'il se réfère aux «droits qui découlent des normes économiques et sociales et de celles relatives à l'éducation, la science et la culture, énoncées dans la Charte de l'Organisation des États américains» (\$ 92-93).

La Cour va dans un premier temps analyser les faits de l'espèce au regard du droit de propriété. Comme à son habitude, elle rappelle d'abord la portée de ce droit, dont elle a une conception assez large, en ce qu'il comprend «l'usage et la jouissance des biens, définis comme les choses matérielles appropriables et tous les droits qui peuvent faire partie de la succession d'une personne ${ }^{96}$. Elle précise ensuite que l'expression « droit acquis » désigne les droits qui sont incorporés dans le patrimoine des personnes ${ }^{97}$. Elle réaffirme néanmoins que ce droit n'est pas absolu puisqu'il peut être soumis à des restrictions ${ }^{98}$, à condition qu'elles empruntent les voies légales adéquates et qu'elles respectent les conditions fixées dans ce même article $21^{99}$.

La Cour calque ensuite son raisonnement sur celui qu'elle avait développé dans l'affaire des Cinq retraités ${ }^{100}$, dont les faits étaient similaires ( $\$ 85-91)$. Elle estime en effet qu'à partir du moment où une personne commence à cotiser, elle acquiert le droit de percevoir une retraite, dans les conditions prévues par la loi, droit qui a des effets patrimoniaux ${ }^{101}(\$ 85)$. Le droit à une retraite est donc qualifié de "droit acquis», reconnu par le droit interne péruvien, qui a fait l'objet, d'après les juridictions internes, d'une réduction illégale. Dès lors, le fait que l'État n'ait pas respecté les décisions juridictionnelles le rend responsable d'une violation du droit à la propriété des victimes $(\$ 88)$.

L'invocation de l'article 26, ensuite, est le moyen pour la Cour de répondre aux critiques doctrinales ${ }^{102}$ dont elle a fait l'objet suite à l'affaire des Cinq retraités ${ }^{103}$. Elle avait en effet refusé de considérer que la diminution des retraites des victimes entraînait une violation de cet article, dans la mesure où il ne consacre que le « développement progressif» de ces droits, qui devait, selon la Cour, être «mesuré en fonction [...] de l'ensemble de la population [...] non pas en fonction de la situation d'un groupe très limité ». La doctrine reprochait alors à la Cour de ne pas

92. Cour IDH, Apitz Barbera et autres c. Venezuela, $\$ 206$.

93. Cour IDH, Yatama c. Nicaragua, exceptions préliminaires, fond, réparations et coûts, 23 juin 2005, série C, $\mathrm{n}^{\circ}$ 127, $\$ 195$.

94. Cour IDH, Acevedo Buendía et autres ("Cesantes y Jubilados de la Contraloría») c. Pérou, exceptions préliminaires, fond, réparations et coûts, $1^{\mathrm{er}}$ juillet 2009, série $\mathrm{C}, \mathrm{n}^{\mathrm{0}} 198$, ci-après «affaire Acevedo Buendía et autres $c$. Pérou ».

95. Droit reconnu et protégé par les juridictions internes mais non effectif, puisque suite à la condamnation de l’État par la décision du Tribunal constitutionnel en date du 21 octobre 1997, ce dernier ne s'est pas conformé à cette décision et n’a donc pas versé les sommes dues au requérant.

96. Cour IDH, Ivcher Bronstein c. Pérou, fond, réparations et coûts, 6 février 2001, série C, $\mathrm{n}^{\circ} 74, \S 120-122$.

97. Cour IDH, Salvador Chiriboga c. Équateur, exceptions préliminaires et fond, 6 mai 2008, série C, nº 179, $\$ 55$.

98. Cour IDH, Ivcher Bronstein, $\$ 128$.

99. Cour IDH, Salvador Chiriboga, \$54.

100. Cour IDH, Cinq retraités c. Pérou, fond, réparations et coûts, 28 février 2003, série C, $\mathrm{n}^{\circ} 98$.

101. Ibid., $\$ 103$.

102. Tara J. Melish, «A pyrrhic victory for Peru's pensioners: pensions, property, and the perversion of progressivity », Revista Cejil, $\mathrm{n}^{\circ}{ }^{1}, 2005, \mathrm{p} .51-65$. Voir également l'opinion concordante du juge Carlos Vicente de Roux Rengifo sous l'arrêt Cour IDH, Cinq retraités c. Pérou.

103. Cour IDH, Cinq retraités c. Pérou. 
reconnaître que ces droits avaient le caractère de « droits subjectifs » et de les soumettre aux obligations générales des articles 1 et 2 de la Convention ${ }^{104}$.

La Cour réaffirme alors en l'espèce «sa pleine compétence pour analyser les violations de tous les droits reconnus dans la Convention américaine» (\$97). Elle fait ensuite référence aux travaux préparatoires de la Convention portant sur cet article 26, et souligne l'intérêt manifesté par les États sur la question d'insérer une référence directe aux droits économiques et sociaux au sein de la Convention, et de leur donner une valeur juridique et un caractère justiciable (\$99). Elle se réfère, enfin, à la place de cette disposition dans l'économie générale de la Convention, l'article 26 étant situé au sein du chapitre III de la partie I, intitulée Des obligations des États et des droits protégés, et par conséquent soumis, tout comme le chapitre II relatif aux droits civils et politiques, au chapitre I relatif aux obligations générales (art. 1 et 2 de la Convention) ${ }^{105}$.

La soumission de cette disposition aux articles 1 et 2 de la Convention permet donc à la Cour de mettre un terme à la polémique et d'affirmer clairement à la fois son caractère normatif, au même titre que les droits contenus dans le chapitre II, mais également le caractère indivisible des droits civils et politiques et des droits économiques, sociaux et culturels. Pour justifier cette démarche, la Cour s'appuie sur divers précédents au niveau international, à commencer par la jurisprudence de la Cour européenne des Droits de l'homme et son célèbre arrêt Airey ${ }^{106}$, qu' elle cite pour rappeler l'interdépendance des droits, les droits civils et politiques ayant « des prolongements économiques et sociaux» $(\$ 101)$. Elle se réfère ensuite à l'observation générale $\mathrm{n}^{\mathrm{o}} 3 \mathrm{du}$ Comité des droits économiques, sociaux et culturels des Nations unies, en ce qu'elle impose à l'État de respecter ses obligations internationales en la matière ${ }^{107}$, et affirme la justiciabilité de ce type de droits $(\$ 102)$.

La Cour consacre ensuite le devoir de «nonrégression » comme corollaire de la progressivité et se fonde encore une fois sur les observations du Comité des droits économiques, sociaux et culturels des Nations unies ${ }^{108}$, mais également sur les décisions de la Commission américaine ${ }^{109}$, et conclut finalement que le caractère régressif peut être invoqué devant elle, afin qu'elle constate une violation de l'article 26 ( $\$ 103$ ).

Néanmoins, même si la Cour tente de désamorcer les critiques sur le plan des principes, leur application au cas d'espèce révèle de nombreuses failles, qui ne feront peut-être que les raviver. En effet, la Cour estime que cette disposition n'est pas violée, en se fondant uniquement sur le fait que le Tribunal constitutionnel interne a annulé les dispositions litigieuses, alors même que l'État a refusé de s'y conformer et donc de restituer aux victimes les sommes dues.

Cette position permet tout d'abord à la Cour d'éviter de se prononcer sur l'existence d'un droit à une retraite reconnu par la Convention. En effet, la Cour ne parle ici de ce droit qu'en ce qu'il est «reconnu par le droit interne ». Dès lors, la Cour ne tire pas de l'article 26 un quelconque droit à la retraite qui lui serait «inhérent », mais semble uniquement affirmer qu'il est justiciable devant elle du fait de sa consécration en droit interne. Ce qui aurait donc pu être sanctionné est bien le maintien des décrets litigieux en droit interne, aboutissant à une régression de ce droit préalablement consacré en droit péruvien. Or le Tribunal constitutionnel ayant déclaré «inapplicables et inconstitutionnels» lesdits décrets, ceux-ci disparaissent de l'ordre juridique en péruvien, et «l'effet cliquet» est préservé. La Cour estime par conséquent qu'il n'existe aucune controverse sur le point de savoir si «les victimes ont droit à un niveau de retraite ou si ce droit a été affecté par l'application injustifiée des décrets mentionnés ", alors que ces derniers ont cessé d'exister $(\$ 107)$.

Néanmoins, si cette consécration du droit à une retraite - et sa préservation - par le droit interne péruvien permettent à la Cour d'affirmer que l'État a rempli son obligation de reconnaître de manière progressive les droits économiques, sociaux et culturels, elle ne considère pas que le refus de l'État de rendre effectif ce droit à l'égard des victimes en serait une restriction, voire une violation. En effet, l'État n'a pas respecté l'obligation qui lui avait été faite par les juridictions internes de restituer aux victimes la différence de montant de leur retraite pour la période durant laquelle les lois et décrets litigieux leur ont été appliqués. Par conséquent, même si la Cour ne fait plus référence de manière explicite au caractère " collectif» de ces droits économiques, sociaux et culturels, c'est donc bien uniquement ce volet qu' elle protège. Des individus ne peuvent pas se fonder sur cet article pour dénoncer le non-respect de leur droit individuel à une retraite, bien que consacré mais non respecté par l'État.

La Cour estime alors que cet aspect est de nature à violer uniquement l'article 21 relatif au droit de propriété, mais également l'article 25 relatif au droit à une

104. Affaire Acevedo Buendía et autres c. Pérou, $\$ 147$.

105. Elle répond ainsi directement à la critique de T. J. Melish, qui reprochait à la Cour de déduire des obligations étatiques différentes au regard du contenu substantiel des droits protégés, fondé uniquement sur la place formelle de ces articles au sein de la Convention, T. J. Melish, «A pyrrhic victory...", p. 64-65.

106. Cour EDH, Airey c. Irlande, 9 octobre 1979, requête n ${ }^{\circ} 6289 / 73, \S 26$.

107. Nations unies, Comité des droits économiques, sociaux et culturels, observation générale $\mathrm{n}^{\circ}$ 3, La nature des obligations des États parties, U.N. Doc. E/ 1991/23, 1990, \$9.

108. Ibid.

109. Pour apprécier si une mesure régressive est compatible avec la Convention américaine, la Commission avait cherché à « déterminer si elle est justifiée par des raisons suffisamment conséquentes ", Commission IDH, Association nationale des anciens employés de l'Institut péruvien de sécurité sociale et autres c. Pérou, 27 mars 2009, Rapport n 38/09, \$140-147. 
protection judiciaire. La Cour considère en effet qu'il existe une « différence de nature » entre ces deux dispositions d'un côté et l'article 26 de l'autre. Ce dernier, «bien que complémentaire » aux premières, n’impose pas aux États les mêmes obligations : «la nature de l'engagement de l'État découlant de l'article 26 de la Convention consiste en l'adoption de mesures [...] - soit par le biais des recours disponibles, soit par la voie législative ou tout autre moyen - permettant d'assurer progressivement la pleine réalisation de certains droits économiques, sociaux et culturels» $(\$ 105)$.

L'État ayant, du fait de cette décision du Tribunal constitutionnel, bien rempli cette obligation, «l'échec» de la mise en œuvre dans le cas concret n'affecte que les droits civils et politiques $(\$ 106)$. Cette solution s'avère dès lors toujours critiquable du point de vue de l'indivisibilité des droits, puisque même si la Cour affirme la justiciabilité de l'article 26, soumis au même titre que les droits civils et politiques aux articles 1 et 2, elle n'impose à l'État que la mise en place d'une reconnaissance progressive de ces droits au niveau interne, sans leur imposer leur respect effectif. L'on observe donc bien toujours une différence de régime quant à ces deux types de droits. De plus, comme le relève Tara J. Melish, le fait de se fonder uniquement sur le droit de propriété pour condamner l'État aboutit à une incohérence, le droit à une retraite ne pouvant, selon elle, être examiné sous l'angle combiné des articles 21 et 26 de la Convention, ces deux aspects « constituant les deux faces d'une même pièce » ${ }^{110}$.

Pour conclure sur cette analyse, on relève une tendance majeure selon laquelle la Cour attache de manière quasi systématique une obligation positive procédurale à chaque droit substantiel de la Convention. Le «changement de cap » que le professeur Laurence BurgorgueLarsen avait pressenti ${ }^{111}$ s'est donc bel et bien confirmé en 2009. Or, cette vision était vigoureusement défendue par l'ancienne présidente Cecilia Medina Quiroga, qui a donc su imposer sa doctrine et laisser son empreinte dans la jurisprudence interaméricaine. L'« ère Medina Quiroga » qu'on avait annoncée lors de notre précédente chronique est en effet marquée par cette volonté de donner toute son ampleur aux articles 8 et 25 de la Convention, lus dorénavant de manière complètement autonome, tant l'un vis-à-vis de l'autre ${ }^{112}$, qu'au regard des autres dispositions de la Convention. Il en découle une double obligation d'enquête, l'une émanant du volet procédural de chaque droit substantiel - et du fait de la violation de ce droit -, et celle fondée sur la combinaison des articles 8 , 25 et 1.1 de la Convention, s'attachant cette fois-ci à toute illégalité constatée en droit interne. Reste à savoir si la nouvelle composition de la Cour, et son tout nouveau président, le juge péruvien Diego Garcia-Sayan élu en novembre 2009, et entré en fonction le $1^{\mathrm{er}}$ janvier 2010, perpétreront cette vision.

110. T. J. Melish, «A pyrrhic victory... », p. 65.

111. L. Burgorgue-Larsen, "Commentaire n 25 sous l'arrêt Bámaca Velásquez c. Guatemala", in Les grandes décisions de la Cour interaméricaine des Droits de l'homme, L. Burgorgue-Larsen et A. Úbeda de Torres (éd.), Bruxelles, Bruylant, 2008, p. 751.

112. Les décisions de l'année 2009 font en effet apparaître que la Cour examine les articles 8 et 25 tout aussi bien de manière autonome que de manière combinée. Or cette question était également un sujet de discorde entre Antônio Augusto Cançado Trindade, qui estime que ces deux dispositions sont indissociables, le recours effectif conditionnant, selon lui, l'existence d'un procès équitable, et Cecilia Medina Quiroga qui plaide pour une reconnaissance du «contenu autonome» de chacun de ces droits. Elle estime en effet que ces deux dispositions ont un champ d'application différent, l'article 25 offrant une garantie plus spécifique, alors qu’une lecture combinée systématique aboutirait à «suggérer que la seule norme qui consacre le droit "aux recours" est l'article 25». Voir leurs deux opinions dissidentes sous Cour IDH, López Álvarez c. Honduras, fond, réparations et coûts, $1^{\text {er }}$ février 2006 , série $\mathrm{C}, \mathrm{n}^{\circ} 141$. 
